Publ. RIMS, Kyoto Univ.

18 (1982), 1193-1212

\title{
Equivariant Stable Homotopy Theory and Idempotents of Burnside Rings
}

By

\author{
Shôrô ARAKI*
}

\section{Introduction}

Let $G$ be a finite group throughout the present work. We denote by $A(G)$ the Burnside ring of $G$. The stable G-homotopy theory is a G-homologycohomology theory of $A(G)$-modules and any idempotent of $A(G)$ decomposes it as a direct sum of $G$-homology-cohomology theories. Such a decomposition for $p$-localized case was partly investigated by Kosniowski [13] and tom Dieck [7].

Let $X$ and $Y$ be pointed $G-C W$ complexes. We assume $X$ to be finite. The group of stable $G$-maps from $X$ to $Y$ is denoted by $\tilde{\omega}_{G}^{0}(X: Y)$. We put $\tilde{\omega}_{G}^{\alpha}(X: Y)=\tilde{\omega}_{G}^{0}\left(\Sigma^{V} X: \Sigma^{U} Y\right)$ for $\alpha=U-V \in R O(G)$. We study $e \tilde{\omega}_{G}^{\alpha}(X: Y)$ for each primitive idempotent $e$ of $A(G)$. Denote by $P$ the set of all conjugacy classes of perfect subgroups of $G$. Primitive idempotents of $A(G)$ correspond bijectively with members of $P$, Dress [9]. Denote by $e_{H}$ the primitive idempotent of $A(G)$ corresponding to $(H) \in P$, then

$$
\tilde{\omega}_{G}^{\alpha}(X: Y)=\coprod_{(H) \in P} e_{H} \tilde{\omega}_{G}^{\alpha}(X: Y) .
$$

Let $H$ be a perfect subgroup of $G$. We denote $N=N_{G}(H)$ and $W=N_{G}(H) / H$ for simplicity. The main result of the present work is the following.

Theorem A. There hold the isomorphisms

$$
e_{H} \tilde{\omega}_{G}^{\alpha}(X: Y) \cong \bar{e}_{H} \tilde{\omega}_{N}^{\alpha^{\prime}}(X: Y) \cong \tilde{e}_{\langle 1\rangle} \tilde{\omega}_{W}^{\alpha^{\prime \prime}}\left(X^{H}: Y^{H}\right)
$$

which are $e_{H} A(G)$ - and $\bar{e}_{H} A(N)$-module isomorphisms respecitvely, where $\bar{e}_{H}$ and $\tilde{e}_{\langle 1}$, denote the primitive idempotents of $A(N)$ and $A(W)$ corresponding to $(H)_{N}$ and the trivial perfect subgroup $\{1\}$ of $W$ respecitvely, $\alpha^{\prime}=\operatorname{res}_{N}^{G} \alpha$ and

Communicated by N. Shimada, March 15, 1982.

* Department of Mathematics, Osaka City University, Osaka 558, Japan. 
$\alpha^{\prime \prime}=U^{H}-V^{H}$ for $\alpha^{\prime}=U-V \in R O(N)$.

Corollary B. There hold the ring isomorphisms

$$
e_{H} A(G) \cong \bar{e}_{H} A(N) \cong \tilde{e}_{\langle 1\rangle} A(W) .
$$

Direct proof of this corollary is not difficult. T. Miyata and T. Yoshida communicated to the author relatively short direct proofs of this corollary.

Theorem A hold also for any $G$-homology-cohomology theories defined by $G$-spectra. The $p$-localized version of Theorem $\mathrm{A}$ is also ture. In fact we prove the more generalized version of Theorem A (Theorem 3.6, (3.7) and Theorem 4.7). We obtain Theorem A by specializing $\pi=\{$ all primes $\}$ and the $p$-localized version by $\pi=\{p\}$.

In Section 1 we observe certain relations between primitive idempotnents of $A(G)_{(\pi)}$ and $A(N)_{(\pi)}$, and their behaviors in Mackey double coset formula. The explicit formula (1.2) for primitive idempotents due to Yoshida [17] is essential. In Section 2 we prove an isomorphism theorem (Theorem 2.5) for Mackey functors on the category $\hat{G}$ of finite $G$-sets. In Section 3 we see briefly that stable $G$-homotopy theory provides Mackey functors on $\hat{G}$, then we obtain the first isomorphism of Theorem A (Theorem 3.6 and (3.7)) by applying Theorem 2.5. In Section 4 we construct the fixed-point exact sequences for stable $G$-homotopy theory and prove the second isomorphism of Theorem A (Theorem 4.7).

\section{§1. Idempotents of Burnside Rings}

Let $\hat{G}$ be the category of finite $G$-sets and $G$-maps. The set of all isomorphism classes in $G$ forms a commutative semi-ring $A^{+}(G)$ with addition and multiplication defined by disjoint unions and direct products (with diagonal $G$-actions) respectively. The Burnside ring of $G$, denoted by $A(G)$, is the Grothendieck ring of $A^{+}(G)$. A finite $G$-set $S$ represents an element of $A(G)$, denoted by $[S]$. Then every element of $A(G)$ can be expressed in the form $[S]-[T]$. Every finite $G$-set is expressed uniquely as the disjoint union of $t$; orbits, which implies that $A(G)$ is additively a free $Z$-module with basis $\{[G / L]$; $(L) \in C(G)\}$, where $C(G)$ denotes the set of conjugacy classes of subgroups of $G$. As to the basic properties of $A(G)$ we refer to [8] [9] [10].

Let $\pi$ be a set of primes and $\mathbb{Z}_{(\pi)}$ the subring of $Q$ consisting of all fractions $a / b$ such that $(a, b)=1$ and $b$ is prime to every member of $\pi$. Thus, $\mathbb{Z}_{(\pi)}=\mathbb{Q}$ in case $\pi=\varnothing ; \boldsymbol{Z}_{(\pi)}=\boldsymbol{Z}$ in case $\pi=\{$ all primes $\} ; \boldsymbol{Z}_{(\pi)}=\boldsymbol{Z}_{(p)}$ in case $\pi=\{p\}$, the 
set consisting of a single prime $p$. We write $A_{(\pi)}=A \otimes \mathbb{Z}_{(\pi)}$ for any abelian group $A$. Let $G \geqq L$, a subgroup. The assignment " $S \mapsto\left|S^{L}\right|$ " defines a semiring homomorphism $A^{+}(G) \rightarrow Z$ and induces the ring homomorphism

$$
\phi_{L}: A(G)_{(\pi)} \longrightarrow Z_{(\pi)},
$$

which is important in studying structure of $A(G)_{(\pi)}[8]$ [9] [17]. E.g., $A(G)_{(\pi)}$ $\ni x=0 \Leftrightarrow \phi_{L}(x)=0$ for all $L \leqq G$.

Primitive idempotents of $A(G)_{(\pi)}$ are discussed in [8] [9] [11] [17]. Following [17] we denote by $S^{\pi}(G)$ the minimal normal subgroup of $G$ by which the quotient is a solvable $\pi$-group. $\quad S^{\pi}(G)$ is the uniquely determined characteristic subgroup of $G$ [9]. $G$ is called to be $\pi$-perfect provided $S^{\pi}(G)=G$. When $\pi=\{$ all primes , $\pi$-perfect groups are perfect groups.

$S^{\pi}(G)$ is always $\pi$-perfect as $S^{\pi}\left(S^{\pi}(G)\right)=S^{\pi}(G)$. Let $P_{\pi}$ denote the set of all conjugacy classes of $\pi$-perfect subgroups of $G$. Primitive idempotents of $A(G)_{(\pi)}$ correspond bijectively with members of $P_{\pi}[9]$ [17].

Let $H$ be a $\pi$-perfect subgroup of $G$ and $e_{H}^{\pi}$ the primitive idempotent corresponding to the conjugacy class $(H)$. Put

$$
S_{\pi}(H, G)=\left\{L \leqq G ; S^{\pi}(L)=H\right\}
$$

following [17]. $e_{H}^{\pi}$ is characterized by

$$
\begin{array}{rlrl}
\phi_{L}\left(e_{H}^{\pi}\right)=1 & & \text { if } \quad L \sim S_{\pi}(H, G) \\
& =0 & & \text { otherwise },
\end{array}
$$

where " " means "conjugate to a member of" [8] [9] [17].

Recently an explicit formula for the idempotent $e_{H}^{\pi}$ has been given by Yoshida [17]. (The formula for the case $\pi=\varnothing$ is given also by Gluck [11].) Let $\mu$ be the Möbius function on the subgroup lattice of $G$. For $D \leqq G$ he defines

$$
\lambda(D, H)=\sum_{L \in S_{\pi}(H, G)} \mu(D, L)
$$

and obtains the explicit formula for $e_{H}^{\pi}$ [17], Theorem 3.1, as follows:

$$
e_{H}^{\pi}=\left(1 /\left|N_{G}(H)\right|\right) \cdot \sum_{D \leqq N_{G}(H)}|D| \lambda(D, H)[G / D] .
$$

Let $K \leqq G$. Restricting $G$-actions to $K$ on each finite $G$-set $S$, one obtains the ring homomorphism

$$
\operatorname{res}_{K}^{G}: A(G)_{(\pi)} \longrightarrow A(K)_{(\pi)},
$$

called the restriction homomorphism. Clearly

$$
\phi_{L}\left(\operatorname{res}_{K}^{G} x\right)=\phi_{L}(x)
$$


for $x \in A(G)_{(\pi)}$ and $L \leqq K$. The assignment " $S \mapsto G \times{ }_{K} S$ ", for each finite $K$-set $S$ induces the linear homomorphism

$$
\operatorname{tr}_{K}^{G}: A(K)_{(\pi)} \longrightarrow A(G)_{(\pi)},
$$

called the transfer homomorphism. By definition

$$
\operatorname{tr}_{K}^{G}[K / L]=[G / L] .
$$

There holds the Frobenius formula

$$
\operatorname{tr}_{K}^{G}\left(x \cdot \operatorname{res}_{K}^{G} y\right)=\left(\operatorname{tr}_{K}^{G} x\right) \cdot y
$$

for $x \in A(K)_{(\pi)}$ and $y \in A(G)_{(\pi)}$. $\operatorname{res}_{K}^{G}$ maps idempotents to idempotents (which may be decomposable), whereas $\operatorname{tr}_{K}^{G}$ does not in general. Obviously res $G_{G}^{G}$ $=\operatorname{tr}_{G}^{G}=\mathrm{id}$ for $K=G$.

Let $H$ be a $\pi$-perfect subgroup of $G$ and put $N=N_{G}(H)$, the normalizer of $H$ in $G$. Let $\bar{e}_{H}^{\pi}$ denote the primitive idempotent of $A(N)_{(\pi)}$ corresponding to $(H)_{N}$, the conjugacy class of $H$ in $N$, which we call the central idempotent of $A(N)_{(\pi)}$. It is characterized by

$$
\begin{array}{rlrl}
\phi_{L}\left(\bar{e}_{H}^{\pi}\right)=1 & & \text { if } \quad L \in S_{\pi}(H, N) \\
& =0 & & \text { otherwise }
\end{array}
$$

since $H \triangleleft N$. (Compare with (1.1).) Remark that $S_{\pi}(H, G)=S_{\pi}(H, N)$ and $\lambda(D, H), D \leqq N$, is the same for $G$ and $N$. Since $N_{G}(H)=N_{N}(H)$, we compute by (1.2) as follows:

$$
\begin{aligned}
\operatorname{tr}_{N}^{G} \bar{e}_{H}^{\pi} & =\left(1 /\left|N_{N}(H)\right|\right) \cdot \sum_{D \leqq N_{N}(H)}|D| \lambda(D, H) \cdot \operatorname{tr}_{N}^{G}[N / D] \\
& =\left(1 /\left|N_{G}(H)\right|\right) \cdot \sum_{D \leqq N_{G}(H)}|D| \lambda(D, H)[G / D] \\
& =e_{H}^{\pi},
\end{aligned}
$$

i.e., we obtain

$$
\operatorname{tr}_{N}^{G} \bar{e}_{H}^{\pi}=e_{H}^{\pi} .
$$

$\operatorname{res}_{N}^{G} e_{H}^{\pi}$ is an idempotent of $A(N)_{(\pi)}$ and we see easily by (1.1) that it decomposes as a sum of primitive idempotents which correspond to conjugacy classes $\left(H^{\prime}\right)_{N}$ in $N$ such that $H^{\prime} \sim H$ in $G$. Such conjugacy classes correspond bijectively to a part of the double cosets $N \backslash G / N$. Let $\left\{g_{1}, \ldots, g_{t}\right\}$ be a complete system of representatives of $N \backslash G / N$. Choose a numeration of this system so that $i \leqq s \Leftrightarrow H_{i}=g_{i} H_{g_{i}}{ }^{-1} \leqq N$ (which does not depend on the choice of the representative $\left.g_{i}\right)$. Then $\left\{\left(H_{\imath}\right)_{N}, 1 \leqq i \leqq s\right\}$ forms the complete set of the above mentioned conjugacy classes $\left(H^{\prime}\right)_{N}$ in $N$. We choose $g_{1}=1$ always, then $H_{1}=H$. 
Let $\bar{e}_{i}^{\pi}$ denote the primitive idempotent of $A(N)_{(\pi)}$ which corresponds to $\left(H_{i}\right)_{N}$. $\bar{e}_{1}^{\pi}=\bar{e}_{H}^{\pi}$, the central idempotent of $A(N)_{(\pi)}$. And we obtain

$$
\operatorname{res}_{N}^{G} e_{H}^{\pi}=\sum_{1 \leqq i \leqq s} \bar{e}_{i}^{\pi}
$$

By (1.4) and (1.5) we see that

$$
\operatorname{res}_{N}^{G} \circ \operatorname{tr}_{G}^{N} \bar{e}_{H}^{\pi}=\sum_{1 \leqq i \leqq s} \bar{e}_{i}^{\pi} .
$$

Next we apply the Mackey decomposition to $\operatorname{res}_{N}^{G} \circ \operatorname{tr}_{N}^{G}$. Putting $N_{i}=N_{G}\left(H_{i}\right)$, $1 \leqq i \leqq t$, we obtain

$$
\operatorname{res}_{N}^{G} \circ \operatorname{tr}_{N}^{G}=\sum_{1 \leqq i \leqq t} \operatorname{tr}_{N \cap N_{\imath}}^{N} \circ \operatorname{res}_{N \cap N_{\imath}}^{N_{2}} \circ C_{i}^{*},
$$

where $c_{i}^{*}: A(N)_{(\pi)} \rightarrow A\left(N_{i}\right)_{(\pi)}$, the isomorphism induced by the conjugation isomorphism $N_{i} \simeq N$ with respect to $g_{i}^{-1}$.

We observe $\operatorname{tr}_{N \cap N_{\imath}}^{N} \circ \operatorname{res}_{N \cap N_{\imath}}^{N_{\imath}}{ }^{\circ} c_{i}^{*}\left(\ddot{e}_{H}^{\pi}\right)$ for each $i, 1 \leqq i \leqq t . \quad c_{i}^{*}$ maps primitive idempotents to primitive ones. By (1.3) we see that

$$
\begin{aligned}
\phi_{L}\left(c_{i}^{*}\left(\bar{e}_{H}^{\pi}\right)\right) & =1 & & \text { if } L \in S_{\pi}\left(H_{i}, N_{i}\right) \\
& =0 & & \text { otherwise. }
\end{aligned}
$$

Thus $c_{i}^{*}\left(\bar{e}_{H}^{\pi}\right)$ is the central idempotent of $A\left(N_{i}\right)_{(\pi)}$. Then

$$
\begin{aligned}
\phi_{L}\left(\operatorname{res}_{N \cap N_{\iota}}^{N_{2}} \circ c_{i}^{*}\left(\bar{e}_{H}^{\pi}\right)\right) & =1 & & \text { if } L \in S_{\pi}\left(H_{i}, N \cap N_{i}\right) \\
& =0 & & \text { otherwise, }
\end{aligned}
$$

which shows that $\operatorname{res}_{N \cap N_{t}}^{N_{i}} \circ c_{i}^{*}\left(\bar{e}_{H}^{\pi}\right)=0$ for $i>s$ and $=$ the central idempotent of $A\left(N_{N}\left(H_{i}\right)\right)_{(\pi)}$ for $1 \leqq i \leqq s$ by $(1.3)$ as $N \cap N_{i}=N_{N}\left(H_{i}\right)$. Let $\overline{\bar{e}}_{i}^{\pi}$ denote the central idempotent of $A\left(N_{N}\left(H_{i}\right)\right)_{(\pi)}$. We have obtained

$$
\begin{aligned}
\operatorname{res}_{N \cap N_{i}}^{N_{i}}{ }^{\circ} c_{i}^{*}\left(\bar{e}_{H}^{\pi}\right) & =\bar{e}_{i}^{\pi} & \text { for } & 1 \leqq i \leqq s \\
& =0 & \text { for } & s<i \leqq t .
\end{aligned}
$$

Apply (1.4) for the pair $\left(N, H_{i}\right)$ and obtain

$$
\operatorname{tr}_{N \cap N_{i}}^{N}\left(\bar{e}_{i}^{\pi}\right)=\bar{e}_{i}^{\pi} \quad \text { for } 1 \leqq i \leqq s .
$$

We add two remarks. Since $\bar{e}_{i}^{\pi}$ is the primitive idempotent of $A\left(N \cap N_{i}\right)_{(\pi)}$ corresponding to $\left(H_{\imath}\right)_{N \cap N_{i}}$, we have the decomposition

$$
\operatorname{res}_{N \cap N_{i}}^{N} \bar{e}_{i}^{\pi}=\overline{\bar{e}}_{i}^{\pi}+\cdots
$$

into primitive ones for $1 \leqq i \leqq s$ by (1.5). Thus

$$
\left(\operatorname{res}_{N \cap N_{i}}^{N} \bar{e}_{i}^{\pi}\right) \cdot \overline{\bar{e}}_{i}^{\pi}=\overline{\bar{e}}_{i}^{\pi} \quad \text { for } 1 \leqq i \leqq s .
$$

The second remark is that $g_{1}=1, H_{1}=H$ and $N_{1}=N$ by our choice. Thus 


$$
\operatorname{tr}_{N \cap N_{1}}^{N}=\operatorname{res}_{N \cap N_{1}}^{N_{1}}=c_{1}^{*}=\mathrm{id} .
$$

\section{§2. Idempotents and Mackey Functors}

Dress [10], Section 4, defined the Burnside functor on $\hat{G}$. Let $T$ be a finite $G$-set and $\hat{G} / T$ the category of objects over $T$. The set of all isomorphism classes of $\hat{G} / T$ forms a commutative semi-ring $A_{G}^{+}(T)$ with addition and multiplication defined by disjoint unions and pull-backs. Its Grothendieck ring is denoted by $A_{G}(T)$. The element of $A_{G}(T)$ represented by an object $f: S \rightarrow T$ of $\hat{G} / T$ is denoted by $[f: S \rightarrow T]$. The Burnside functor $A_{G}=\left(A_{G *}, A_{G}^{*}\right)$ on $\hat{G}$ is a pair of functors $A_{G *}: \hat{G} \rightarrow \mathbf{A b}$ and $A_{G}^{*}: \hat{G}^{o p} \rightarrow \mathbf{A b}$ such that $A_{G *}(T)$ $=A_{G}^{*}(T)=A_{G}(T)$ on each object $T$ and, for a morphism $f: S \rightarrow T$ in $\hat{G}, A_{G *}(f)$ $=f_{*}: A_{G}(S) \rightarrow A_{G}(T)$ is given by $f_{*}[g: U \rightarrow S]=[f \circ g: U \rightarrow T]$ and $A_{G}^{*}(f)=f^{*}$ : $A_{G}(T) \rightarrow A_{G}(S)$ by $f *[h: W \rightarrow T]=\left[W \times{ }_{T} S \rightarrow S\right]$.

As for the definition of a Mackey functor $M=\left(M_{*}, M^{*}\right)$ on $\hat{G}$ we refer to [7], p. 68. The Burnside functor $A_{G}$ is a Mackey functor on $\hat{G}$. Moreover, $f^{*}$ is multiplicative (i.e., $A_{G}^{*}$ is ring-valued) and there holds the Frobenius property among $f_{*}, f^{*}$ and multiplication, i.e., $A_{G}$ is a Green functor in the sense of [10].

There holds the canonical isomorphism

$$
A_{G}(G / K) \simeq A(K)
$$

for $K \leqq G$ such that

$$
p_{*}=\operatorname{tr}_{L}^{K} \quad \text { and } \quad p^{*}=\operatorname{res}_{L}^{K}
$$

for $L \leqq K \leqq G$ and $p: G / L \rightarrow G / K$, the canonical projection.

Let $M=\left(M_{*}, M^{*}\right)$ be any Mackey functor on $\hat{G}$. We write $M_{*}(f)=f_{*}$ and $M^{*}(f)=f^{*}$ for a morphism $f: S \rightarrow T$ in $\hat{G} . \quad M(T)$ becomes an $A_{G}(T)$-module by $[f: S \rightarrow T] \cdot x=f_{*} \circ f^{*} x, x \in M(T),[7][10]$. By these module actions $M$ is an $A_{\mathrm{G}}$-module in the sense that $M^{*}$ is a module-valued functor $\left(f^{*}(x y)=\left(f^{*} x\right)\left(f^{*} y\right)\right.$ for $f: S \rightarrow T, x \in A_{G}(T)$ and $\left.y \in M(T)\right)$ and there holds the Frobenius property among $f_{*}, f^{*}$ and module action [10], Proposition 4.2. We write $p_{*}=\operatorname{tr}_{L}^{K}$, $p^{*}=\operatorname{res}_{L}^{K}$ for any Mackey functor $M, L \leqq K \leqq G$ and $p: G / L \rightarrow G / K$, the canonical projection, in conformity with the above mentioned identities for $A_{G}$.

Let $\pi$ be a set of primes and $M$ a $\boldsymbol{Z}_{(\pi)}$-module-valued Mackey functor. Put $A_{G, \pi}=A_{G} \otimes Z_{(\pi)}$. The above module action of $A_{G}$ on $M$ makes $M$ an $A_{G, \pi}$-module. 
For each $K \leqq G, M(G / K)$ is an $A(K)_{(\pi)}$-module. Hence primitive idempotents of $A(K)_{(\pi)}$ decomposes $M(G / K)$ as a direct sum of submodules. In particular

$$
M(p t)=\coprod_{(H) \in P_{\pi}} e_{H}^{\pi} M(p t) .
$$

We observe $e_{H}^{\pi} M(p t)$ as an $e_{H}^{\pi} A(G)_{(\pi)}$-module.

Let $H$ be a $\pi$-perfect subgroup of $G$ and $N=N_{G}(H)$. Let $\bar{e}_{H}^{\pi}$ be the central idempotent of $A(N)_{(\pi)}$. We want to discuss $\operatorname{res}_{N}^{G} \circ \operatorname{tr}_{N}^{G}\left(\bar{e}_{H}^{\pi} x\right)$ for $\bar{e}_{H}^{\pi} x \in \bar{e}_{H}^{\pi} M(G / N)$. The axiom (M1) for the Mackey functor [7] applied to the pull-back diagram

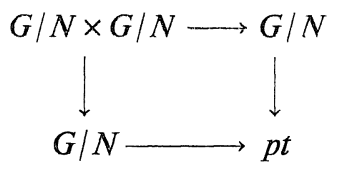

implies the Mackey decomposition

$$
\operatorname{res}_{N}^{G} \circ \operatorname{tr}_{N}^{G}=\sum_{1 \leqq i \leqq t} \operatorname{tr}_{N \cap N_{i}}^{N} \circ \operatorname{res}_{N \cap N_{\imath}}^{N_{i}} \circ c_{i}^{*}
$$

for $M[10][12]$ (the same formula as (1.6)), where we used the same notations as in Section 1, i.e., $\left\{g_{1}, \ldots, g_{t}\right\}\left(g_{1}=1\right)$ is a complete system of representatives of $N \backslash G / N, N_{i}=g_{i} N g_{i}^{-1}$, and $c_{i}^{*}: M(G / N) \simeq M\left(G / N_{i}\right)$, the isomorphism induced by the right multiplication with $g_{i}: G / N_{i} \simeq G / N$, for $1 \leqq i \leqq l$.

Put

$$
\bar{x}_{i}=\operatorname{res}_{N \cap N_{i}}^{N_{2}}\left(c_{i}^{*} x\right) \in M\left(G / N \cap N_{i}\right), 1 \leqq i \leqq t .
$$

As res ${ }_{N \cap N_{i}}^{N_{i}}$ and $c_{i}^{*}$ preserve module actions we see that

$$
\begin{aligned}
& \operatorname{res}_{N \cap N_{i}}^{N_{i}} \mathcal{C}_{i}^{*}\left(\bar{e}_{H}^{\pi} x\right)=\bar{e}_{i}^{\pi} \bar{x}_{i} \quad \text { for } \quad 1 \leqq i \leqq s, \\
& =0 \text { for } s<i \leqq t
\end{aligned}
$$

by (1.7). Next we put

$$
x_{i}=\operatorname{tr}_{N \cap N_{i}}^{N}\left(\bar{e}_{i}^{\pi} \bar{x}_{i}\right) \in M(G / N), \quad 1 \leqq i \leqq s .
$$

Then

$$
\operatorname{tr}_{N \cap N_{i}}^{N}\left(\bar{e}_{i}^{\pi} \bar{x}_{i}\right)=\operatorname{tr}_{N \cap N_{i}}^{N}\left(\left(\operatorname{res}_{N \cap N_{i}}^{N} \bar{e}_{i}^{\pi}\right) \overline{\bar{e}}_{i}^{\pi} \bar{x}_{i}\right)=\bar{e}_{i}^{\pi} x_{i}, \quad 1 \leqq i \leqq s,
$$

by (1.9). For $i=1$, the remark (1.10) is applicable also for $M$ and we see that

$$
\bar{e}_{1}^{\pi} x_{1}=\bar{e}_{H}^{\pi} x,
$$

the given element. Thus we obtain 
Proposition 2.1. Using the notations of Section 1 we have the direct sum decomposition

$$
\left(\operatorname{res}_{N}^{G} e_{H}^{\pi}\right) M(G / N)=\coprod_{1 \leqq i \leqq s} \bar{e}_{i}^{\pi} M(G / N),
$$

and, for any $\bar{e}_{H}^{\pi} x \in \bar{e}_{H}^{\pi} M(G / N)$, we have the decomposition

$$
\operatorname{res}_{N}^{G} \circ \operatorname{tr}_{N}^{G}\left(\bar{e}_{H}^{\pi} x\right)=\sum_{1 \leqq i \leqq s} \bar{e}_{i}^{\pi} x_{i}
$$

such that

$$
\bar{e}_{i}^{\pi} x_{i}=\operatorname{tr}_{N \cap N_{i}}^{N} \circ \operatorname{res}_{N \cap N_{i}}^{N_{i}} \circ c_{i}^{*}\left(\bar{e}_{H}^{\pi} x\right)
$$

and

$$
\bar{e}_{1}^{\pi} x_{1}=\bar{e}_{H}^{\pi} x \text {, the given element }
$$

Put

$$
\operatorname{tr}_{N}^{\prime G}=\operatorname{tr}_{N}^{G} \mid \bar{e}_{H}^{\pi} M(G / N): \bar{e}_{H}^{\pi} M(G / N) \longrightarrow e_{H}^{\pi} M(p t) .
$$

Suppose $\bar{e}_{H}^{\pi} x \in \operatorname{Ker} \operatorname{tr}_{N}^{\prime G}$. Then $\operatorname{res}_{N}^{G \circ \operatorname{tr}}{ }_{N}^{G}\left(\bar{e}_{H}^{\pi} x\right)^{\circ}=\sum_{1 \leqq i \leqq s} \bar{e}_{i}^{\pi} x_{i}=0$. Hence $\bar{e}_{i}^{\pi} x_{i}=0$ for all $i, 1 \leqq i \leqq s$. In particular $\bar{e}_{H}^{\pi} x=\bar{e}_{1}^{\pi} x_{1}=0$. Thus we obtain

$$
\operatorname{tr}_{N}^{\prime G}: \bar{e}_{H}^{\pi} M(G / N) \longrightarrow e_{H}^{\pi} M(p t) \text { is monomorphic. }
$$

Let

$$
\operatorname{res}_{N}^{\prime G}: e_{H}^{\pi} M(p t) \longrightarrow \bar{e}_{H}^{\pi} M(G / N)
$$

be the $e_{H}^{\pi} A(G)_{(\pi)}$-module map defined by

$$
\operatorname{res}_{N}^{\prime G}(x)=\bar{e}_{H}^{\pi} \cdot \operatorname{res}_{N}^{G} x, x \in e_{H}^{\pi} M(p t) .
$$

By Frobenius property and (1.4) we see that

$$
\operatorname{tr}_{N}^{\prime G} \operatorname{res}_{N}^{\prime G}(x)=\operatorname{tr}_{N}^{G}\left(\bar{e}_{H}^{\pi} \cdot \operatorname{res}_{N}^{G} x\right)=e_{H}^{\pi} x=x
$$

for $x \in e_{H}^{\pi} M(p t)$. Thus

$$
\operatorname{tr}_{N}^{\prime G} \circ \operatorname{res}_{N}^{\prime G}=\mathrm{id}
$$

which shows that $\operatorname{tr}_{N}^{\prime G}$ is epimorphic and hence isomorphic by (2.3). Clearly res $_{N}^{\prime}$ is the inverse to $\operatorname{tr}_{N}^{\prime G}$ and we obtain

Theorem 2.5. Let $\pi$ be a set of primes, $M$ a $Z_{(\pi)^{-}}$-module-valued Mackey functor on $G, H$ a $\pi$-perfect subgroup of $G$ and $N=N_{G}(H)$. Let $e_{H}^{\pi}$ be the primitive idempotent of $A(G)_{(\pi)}$ corresponding to $(H) \in P_{\pi}$ and $\bar{e}_{H}^{\pi}$ the central idempotent of $A(N)_{(\pi)}$. Then there holds the $e_{H}^{\pi} A(G)_{(\pi)}$-module isomorphism

$$
\operatorname{res}_{N}^{\prime G}: e_{H}^{\pi} M(p t) \cong \bar{e}_{H}^{\pi} M(G / N) \text {. }
$$




\section{§3. Stable $\mathbb{G}$-Homotopy Theory}

By a $G$-module $V$ we mean a finite dimensional real or complex $G$-module equipped with an invariant metric for simplicity. By $S^{V}$ and $B^{V}$ we denote the unit sphere and unit ball of $V$ respectively. We put $\Sigma^{V}=B^{V} / S^{V}$, which is $G$ homeomorphic to the one-point compactification of $V$.

Let $X$ and $Y$ be pointed $G$-CW complexes. We assume $X$ to be finite. By the group of stable-G-maps from $X$ to $Y$ we understand

$$
\tilde{\omega}_{G}^{0}(X: Y)=\operatorname{colim}\left[\Sigma^{V} X, \Sigma^{V} Y\right]^{G}
$$

[8], Section 7, where $[,]^{G}$ denotes the set of $G$-homotopy classes of pointed $G$-maps, $\Sigma^{V} X=\Sigma^{V} \wedge X, V$ runs over the system of complex $G$-modules which is directed by $G$-embeddings as $G$-submodules, and the colimit is taken with respect to suspensions

$$
\Sigma_{*}^{W}:\left[\Sigma^{V} X, \Sigma^{V} Y\right]^{G} \longrightarrow\left[\Sigma^{W \oplus V} X, \Sigma^{W \oplus V} Y\right]^{G} .
$$

$\tilde{\omega}_{G}^{0}(X: Y)$ is a well-defined abelian group.

We use complex $G$-modules by the following two reasons: i) the directed system of complex $G$-modules may be regarded as a cofinal subsystem of that of real $G$-modules so that we loose nothing by this restriction; ii) the group of complex automorphisms of a complex $G$-module $V$ is connected so that $G$-maps $\Sigma^{V} \rightarrow \Sigma^{V}$ induced by complex automorphisms of $V$ are all $G$-homotopic to the identity, which makes several identifications among $G$-homotopy sets coming from isomorphisms of $G$-modules unique.

Let $f: S \rightarrow T$ be a map in $\widehat{G}$. Endowing discrete topology to $S$ and $T$ respectively, a $G$-embedding $i: S \subset T \times V$ such that $V$ is a complex $G$-module and $\operatorname{pr}_{1} \circ i=f$ is called an admissible embedding for $f$. The existence of an admissible embedding is easily shown by making use of the complex permutation representation $V_{S}$ of $S$. Let $i: S \subset T \times V$ be an admissible embedding for $f$. We may assume that $i(S) \subset T \times \operatorname{Int} B^{V}$. Regard $S$ and $T$ as 0-dimensional $G$ manifolds and let $v i$ be the normal $G$-bundle of the embedding $i$. Then $v i$ $\simeq{ }_{G} S \times V$. Choose the normal disk $G$-bundle $D v i$ so that $D v i \subset T \times B^{V}$. Since $D v i \simeq{ }_{G} S \times B^{V}$, the Thom construction gives a pointed $G$-map

$$
\operatorname{tr} f: T^{+} \wedge \Sigma^{V} \longrightarrow S^{+} \wedge \Sigma^{V}
$$

This construction is of course a very special case of the equivariant Becker- 
Gottlieb transfer [15]. (Compare also with [8], §7, in which the case of compact Lie group actions is discussed.) The following properties of $\operatorname{tr} f$ are easily shown by standard techniques and left to readers.

(3.1) The stable class $\{\operatorname{tr} f\} \in \tilde{\omega}_{G}^{0}\left(T^{+}: S^{+}\right)$is uniquely determined by $f$.

(3.2) Let $f: S_{1} \rightarrow S_{2}$ and $g: S_{2} \rightarrow S_{3}$ be morphisms in $G$. Then

$$
\{\operatorname{tr}(g \circ f)\}=\{\operatorname{tr} f\} \circ\{\operatorname{tr} g\}
$$

as stable G-maps.

(3.3) Let

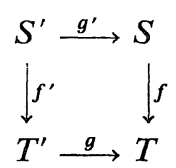

be a pull-back diagram in $\hat{G}$. Then

$$
\left\{g^{\prime+}\right\} \circ\{\operatorname{tr} f\}=\{\operatorname{tr} f\} \circ\left\{g^{+}\right\}
$$

as stable G-maps.

We define a bifunctor

$$
\omega_{G}[X: Y]: \hat{G} \longrightarrow \mathbf{A b}
$$

as follows:

$$
\omega_{G}[X: Y](S)=\tilde{\omega}_{G}^{0}\left(S^{+} \wedge X: Y\right)
$$

on objects; for a morphism $f: S \rightarrow T$ in $\hat{G}$ we put

$$
f_{*}=(\operatorname{tr} f \wedge 1)^{*}: \tilde{\omega}_{G}^{0}\left(S^{+} \wedge X: Y\right) \longrightarrow \tilde{\omega}_{G}^{0}\left(T^{+} \wedge X: Y\right)
$$

which gives a covariant functor by (3.2), and

$$
f^{*}=\left(f^{+} \wedge 1\right)^{*}: \tilde{\omega}_{G}^{0}\left(T^{+} \wedge X: Y\right) \longrightarrow \tilde{\omega}_{G}^{0}\left(S^{+} \wedge X: Y\right)
$$

which gives obviously a contravariant functor.

Proposition 3.4. $\omega_{G}[X: Y]$ is a Mackey functor.

Proof. (3.3) implies the axiom (M1) of [7], p. 68. As to the axiom (M2), let $S \Perp T$ be a disjoint union of finite $G$-sets, then $(S \Perp T)^{+}=S^{+} \vee T^{+}$and

$$
\begin{gathered}
\tilde{\omega}_{G}^{0}\left((S \Perp T)^{+} \wedge X: Y\right)=\tilde{\omega}_{G}^{0}\left(\left(S^{+} \wedge X\right) \vee\left(T^{+} \wedge X\right): Y\right) \\
\simeq \tilde{\omega}_{G}^{0}\left(S^{+} \wedge X: Y\right) \oplus \tilde{\omega}_{G}^{0}\left(T^{+} \wedge X: Y\right) .
\end{gathered}
$$

Let $L \leqq G$. Since the directed system of $L$-modules which are obtained 
from $G$-modules by restriction of actions is a cofinal subsystem of that of arbitrary $L$-modules, we get the homomorphism

$$
\psi_{L}^{G}=\operatorname{res}_{L}^{G}: \tilde{\omega}_{G}^{0}(X: Y) \longrightarrow \tilde{\omega}_{L}^{0}(X: Y)
$$

by restricting $G$-actions to $L$-actions. On the other hand we get the isomorphism

$$
\kappa: \tilde{\omega}_{G}^{0}\left((G / L)^{+} \wedge X: Y\right) \simeq \tilde{\omega}_{L}^{0}(X: Y)
$$

by restricting stable $G$-maps to $\{L\}^{+} \wedge X \simeq_{L} X$, which we regard as the canonical isomorphism. Let

$$
p: G / L \longrightarrow p t
$$

be the unique $G$-map. We can easily identify

$$
p^{*}=\operatorname{res}_{L}^{G}
$$

via the canonical isomorphism $\kappa$. We define

$$
\operatorname{tr}_{L}^{G}=p_{*} \circ \kappa^{-1}: \tilde{\omega}_{L}^{0}(X: Y) \longrightarrow \tilde{\omega}_{G}^{0}(X: Y) .
$$

With these setting we apply Theorem 2.5 to the Mackey functor $\omega_{G}[X: Y]$ and obtain

Theorem 3.5. Let $X$ and $Y$ be pointed G-CW complexes. Assume $X$ to be finite. Let $\pi$ be a set of primes. Using the same notations as in Theorem 2.5 there holds the $e_{H}^{\pi} A(G)_{(\pi)}$-module isomorphism

$$
\operatorname{res}_{N}^{\prime G}: e_{H}^{\pi} \tilde{\omega}_{G}^{0}(X: Y)_{(\pi)} \cong \bar{e}_{H}^{\pi} \tilde{\omega}_{N}^{0}(X: Y)_{(\pi)}
$$

The above theorem applies also to $G$-homology and $G$-cohomology theories. Any G-cohomology theory defined on the category of (finite) G-CW complexes satisfying suitable axioms is representable by a $G$-spectrum [2] [14]. So we discuss here only $G$-homology and $G$-cohomology theories defined by $G$-spectra [2] [13]. We use $G$-spectra indexed by complex (virtual) G-modules in the same reason as the definition of the group of stable $G$-maps. Practically we may restrict our $G$-spectra to those indexed by a cofinal subsystem of that of complex $G$-modules and will do so in the sequel.

Let $\rho=\rho_{G}$ be the complex regular representation of $G . \quad\{n \rho: n \in \mathbb{Z}\}$ is one of such cofinal subsystems. We use this system particularly. A G-spectrum $E_{G}=\left\{E_{n}, \varepsilon_{n}: \Sigma^{\rho} E_{n} \rightarrow E_{n+1} ; n \in \mathbb{Z}\right\}$ consists of a pointed $G-C W$ complex $E_{n}$ and a pointed $G$-map (structure map) $\varepsilon_{n}: \Sigma^{\rho} E_{n} \rightarrow E_{n+1}$ for each $n \in \mathbb{Z}$. When $E_{n}$ $=\Sigma^{n \rho}$ and $\varepsilon_{n}=\mathrm{id}: \sum^{\rho} \sum^{n \rho}=\Sigma^{(n+1) \rho}$ for $n \geqq 0\left(E_{n}=p t\right.$ for $\left.n<0\right)$, the $G$-spectrum 
is called the $G$-sphere spectrum and denoted by $\Sigma_{G}$.

Let $E_{G}=\left\{E_{n}, \varepsilon_{n} ; n \in \mathbb{Z}\right\}$ be a $G$-spectrum and $L \leqq G$. As $\operatorname{res}_{L}^{G} \rho_{G}=|G / L| \cdot \rho_{L}$, where $\rho^{\prime}=\rho_{L}$ is the complex regular representation of $L$, putting

$$
\begin{aligned}
& E_{|G / L| n+k}^{\prime}=\Sigma^{k \rho^{\prime}} E_{n} \quad \text { for } \quad 0 \leqq k<|G / L| \\
& {\varepsilon^{\prime}}_{|G / L| n+k}=\text { id } \quad \text { for } 0 \leqq k<|G / L|-1 \\
& =\varepsilon_{n} \quad \text { for } \quad k=|G / L|-1 \text {, }
\end{aligned}
$$

we get an $L$-spectrum

$$
\psi_{L} E_{G}=\left\{E_{n}^{\prime}, \varepsilon_{n}^{\prime} ; n \in \mathbb{Z}\right\}
$$

by restricting $G$-actions to $L$-actions. Clearly

$$
\psi_{L} \Sigma_{G}=\Sigma_{L}
$$

The $E_{G}$-homology-cohomology group in degree 0 (homology with respect to $Y$ and cohomology with respect to $X$ ) is defined by

$$
E_{G}^{0}(X ; Y)=\operatorname{colim}\left[\Sigma^{n \rho} X, E_{n} \wedge Y\right]^{G},
$$

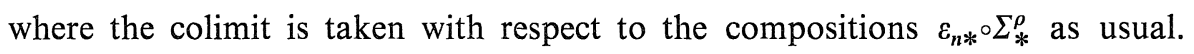
$E_{G}^{O}(X: Y)$ is a well-defined abelian group. Obviously

$$
\Sigma_{G}^{0}(X: Y)=\tilde{\omega}_{G}^{0}(X: Y) .
$$

Again we obtain a Mackey functor $\hat{G} \rightarrow \mathbf{A b}$ by the assignment: $S \mapsto E_{G}^{0}\left(S^{+}\right.$ $\wedge X: Y)$ and " $f: S \rightarrow T^{\prime \prime} \mapsto f_{*}=(\operatorname{tr} f \wedge 1)^{*}$ and $f^{*}=\left(f^{+} \wedge 1\right)^{*}$. Also we have the restriction homomorphism

$$
\psi_{L}^{G}=\operatorname{res}_{L}^{G}: E_{G}^{0}(X: Y) \longrightarrow\left(\psi_{L} E_{G}\right)^{0}(X: Y)
$$

and the transfer homomorphism

$$
\operatorname{tr}_{L}^{G}:\left(\psi_{L} E_{G}\right)^{0}(X: Y) \longrightarrow E_{G}^{0}(X: Y)
$$

together with the canonical isomorphism

$$
\kappa: E_{G}^{0}\left((G / L)^{+} \wedge X: Y\right) \cong\left(\psi_{L} E_{G}\right)^{\circ}(X: Y)
$$

in the parallel way to the case of $\tilde{\omega}_{G}^{0}$.

Now apply Theorem 2.5 to the above Mackey functor and obtain

Theorem 3.6. Under the same assumptions and notations as in Theorem 3.5 there holds the $e_{H}^{\pi} A(G)_{(\pi)}$-module isomorphism

$$
\operatorname{res}_{N}^{\prime G}: e_{H}^{\pi} E_{G}^{0}(X: Y)_{(\pi)} \cong \bar{e}_{H}^{\pi}\left(\psi_{L} E_{G}\right)^{0}(X: Y)_{(\pi)}
$$

for any $G$-spectrum $E_{G}$. 
Let $\alpha \in R O(G)$ and express $\alpha=U-V$ as a difference of real $G$-modules. The $E_{G}$-homology-cohomology group in degree $\alpha$ is defined by

$$
E_{G}^{\alpha}(X: Y)=E_{G}^{0}\left(\Sigma^{V} X: \Sigma^{U} Y\right) .
$$

Let $\alpha=U^{\prime}-V^{\prime}$ be another expression. We can certainly find an additive isomorphism

$$
E_{G}^{0}\left(\Sigma^{V} X: \Sigma^{U} Y\right) \simeq E_{G}^{0}\left(\Sigma^{V^{\prime}} X: \Sigma^{U^{\prime}} Y\right),
$$

but it is no more canonical and there are many choices of this isomorphism. So, as far as we are interested in additive structures we may use the $R O(G)$ grading; but, when we are interested in mulitplicative structure based on ring$G$-spectra, we will meet with serious troubles in $R O(G)$-grading as to commutativity etc., and we need some other device which will be discussed in another occasion.

Anyway we get the restriction homomorphism

$$
\psi_{L}^{G}=\operatorname{res}_{L}^{G}: E_{G}^{\alpha}(X: Y) \longrightarrow\left(\psi_{L} E_{G}\right)^{\psi_{L}^{\alpha}}(X: Y)
$$

and the transfer homomorphism

$$
\operatorname{tr}_{L}^{G}:\left(\psi_{L} E_{G}\right)^{\psi_{L}^{\alpha}}(X: Y) \longrightarrow E_{G}^{\alpha}(X: Y)
$$

in degree $\alpha \in R O(G)$, where $\psi_{L} \alpha=\operatorname{res}_{L}^{G} U-\operatorname{res}_{L}^{G} V \in R O(L)$ for $\alpha=U-V \in R O(G)$.

By the above definition we see that we may apply Theorem 3.6 to $E_{G}^{\alpha}$ and obtain the $e_{H}^{\pi} A(G)_{(\pi)}$-module isomorphism

$$
\operatorname{res}_{N}^{\prime G}: e_{H}^{\pi} E_{G}^{\alpha}(X: Y)_{(\pi)} \cong \bar{e}_{H}^{\pi}\left(\psi_{L} E_{G}\right)^{\psi_{L}^{\alpha}}(X: Y)_{(\pi)} .
$$

\section{§4. Fixed-Point Exact Sequences}

Let $G \triangleright K$, a normal subgroup; then $\left(\rho_{G}\right)^{K}=\rho_{G / K}$, the complex regular representation of $G / K$. Let $E_{G}=\left\{E_{n}, \varepsilon_{n} ; n \in \mathbb{Z}\right\}$ be a $G$-spectrum. Putting

$$
\begin{aligned}
& E_{n}^{\prime \prime}=E_{n}^{K}, \\
& \varepsilon_{n}^{\prime \prime}=\varepsilon_{n}^{K}: \Sigma^{\rho^{\prime \prime}} E_{n}^{\prime \prime} \longrightarrow E_{n+1}^{\prime \prime}, \quad \rho^{\prime \prime}=\rho_{G / K},
\end{aligned}
$$

for $n \in \mathbb{Z}$, we get a $G / K$-spectrum

$$
\phi_{K} E_{G}=\left\{E_{n}^{\prime \prime}, \varepsilon_{n}^{\prime \prime} ; n \in \mathbb{Z}\right\}
$$

which is called the $K$-fixed-point spectrum of $E_{G}$. Clearly

$$
\phi_{K} \Sigma_{G}=\Sigma_{G / K} .
$$


By restriction to $K$-fixed-points we get a homomorphism

$$
\phi_{K}^{G}: E_{G}^{\alpha}(X: Y) \longrightarrow\left(\phi_{K} E_{G}\right)^{\phi_{K}^{\alpha}}\left(X^{K}: Y^{K}\right)
$$

called the $K$-fixed-point homomorphism, where $\phi_{K} \alpha=U^{K}-V^{K} \in R O(G)$ for $\alpha=U-V \in R O(G)$.

We construct an exact sequence involving $\phi_{K}^{G}$ which generalizes the fixedpoint exact sequence for $G=\mathbb{Z} / 2$, [3], Section 1 .

Decompose

$$
\rho_{G}=\rho_{1} \oplus \rho_{2}, \rho_{2}=\rho_{G}^{K} \simeq \rho_{G / K} \quad \text { and } \quad \rho_{1}^{K}=\{0\} .
$$

For each integer $n>0$ we get a $G$-homotopy commutative diagram of pointed $G$-cofibrations

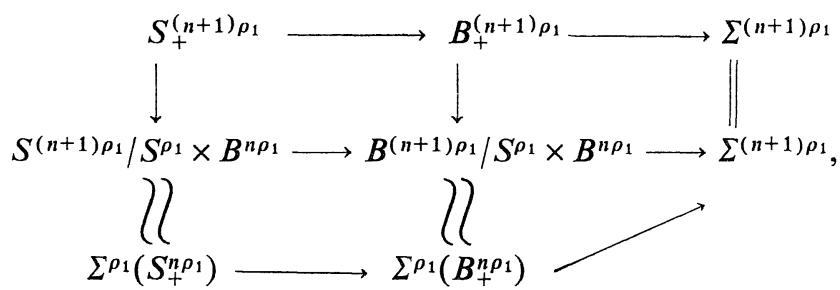

where we identify $B^{(n+1) \rho_{1}}=B^{\rho_{1}} \times B^{n \rho_{1}}, S^{(n+1) \rho_{1}}=\partial\left(B^{\rho_{1}} \times B^{n \rho_{1}}\right)=S^{\rho_{1}} \times B^{n \rho_{1}} U$ $B^{\rho_{1}} \times S^{n \rho_{1}}$, which implies the following commutative diagram with two horizontal exact sequences:

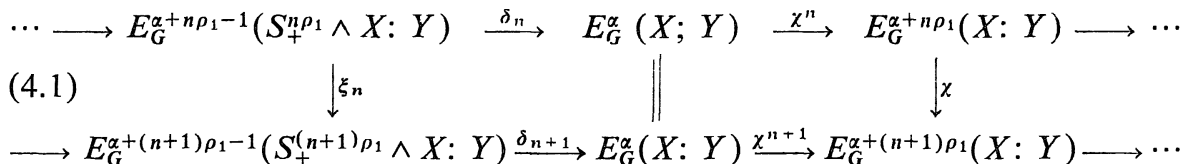

for each $\alpha \in R O(G)$ by fixing the same expression $\alpha=U-V$, where the homomorphism $\chi$ is induced by the inclusion $\chi=\chi_{\rho_{1}}: \Sigma^{0} \subset \Sigma^{\rho_{1}}$ and $\xi_{n}$ is induced by the collapsing map $S_{+}^{(n+1) \rho_{1}} \rightarrow \Sigma^{\rho_{1}}\left(S_{+}^{n \rho_{1}}\right)$. (Compare with the commutative diagram of [3], p. 5.) Take the colimit in vertical direction of this diagram and obtain an exact seqeunce which is an $S$-dual version of the localization exact sequence of tom Dieck [5] under a specified situation. We identify this exact sequence with our desired exact sequence.

\section{Define}

$$
\left(\lambda_{K} E_{G}\right)^{\alpha}(X: Y)=\underset{n}{\operatorname{colim}}\left[E_{G}^{\alpha+n \rho_{1}-1}\left(S_{+}^{n \rho_{1}} \wedge X: Y\right), \xi_{n}\right],
$$

and we prove the isomorphism 


$$
\operatorname{colim}_{n}\left[E_{G}^{\alpha+n \rho_{1}}(X: Y), \chi\right] \cong\left(\phi_{K} E_{G}\right)^{\phi_{K}^{\alpha}}\left(X^{K}: Y^{K}\right) .
$$

First we prove

Lemma 4.4. $\operatorname{colim}_{n}\left[E_{G}^{n \rho_{1}}\left(X / X^{K}: Y\right), \chi\right]=0$.

Proof. Take $x=\{f\} \in \operatorname{colim}\left[E_{G}^{n \rho_{1}}\left(X / X^{K} ; Y\right), \chi\right] . \quad x$ is represented by a $G$-map $f: \Sigma^{m \rho}\left(X / X^{K}\right) \rightarrow \Sigma^{n \rho_{1}} E_{m} \wedge Y$. We want to show that replacing $f$ by another representative $g$ of $x, g^{L} \simeq 0$ for all $L \leqq G$; then $g \simeq{ }_{G} 0$ by [4], Chapter II, Lemma 5.2, and hence $x=0$. Suppose $L \geqq K$, then $p t=\left(X / X^{K}\right)^{K} \supset\left(X / X^{K}\right)^{L}$; thus $\left(X / X^{K}\right)^{L}=p t,\left(\sum^{m \rho}\left(X / X^{K}\right)\right)^{L}=p t \quad$ and $f^{L}=0$. Next, suppose $L \nsupseteq K$. Since $\rho_{G}$ is the complex regular representation of $G$, there exists a non-zero $v \in \rho_{G}$ such that $G_{v}=L$. Let $v=\left(v_{1}, v_{2}\right) \in \rho_{1} \oplus \rho_{2}=\rho_{G}$, then $v_{1} \neq 0$ and $\rho_{1}^{L} \neq\{0\}$. Thus $\left(\Sigma^{k \rho_{1}}\right)^{L}$ is a sphere of dimension $\geqq 2 k$ for any integer $k>0$. In the present colimit $f$ and $\left(\chi^{k} \wedge 1\right) \circ f: \Sigma^{m \rho}\left(X / X^{K}\right) \rightarrow \Sigma^{(k+n) \rho_{1}} E_{m} \wedge Y$ represent the same element $x$ for any integer $k>0$. Since $X$ is finite by our assumption, we may choose $k$ large enough so that $\operatorname{dim} \Sigma^{m}\left(X / X^{K}\right)<2(k+n)-1$. Now, put $g=\left(\chi^{k} \wedge 1\right) \circ f$; $\operatorname{dim}\left(\Sigma^{m \rho}\left(X / X^{K}\right)\right)^{L}<2(k+n)-1$ and $\left(\Sigma^{(k+n) \rho_{1}} E_{m} \wedge Y\right)^{L}$ is at least $(2(k+n)-1)$ connected for any $L \geqq K$; thus $g^{L} \simeq 0$ for all $L \leqq G$ and $g \simeq{ }_{G} 0$.

Proof of (4.3). We prove the case $\alpha=0$. General case follows from this special case by replacing $X$ by $\Sigma^{V} X$ and $Y$ by $\Sigma^{U} Y$ for $\alpha=U-V \in R O(G)$.

Consider the exact sequences associated with the $G$-cofibration $X^{K} \rightarrow X$ $\rightarrow X / X^{K}$ and take the colimit of these sequences with respect to $\chi$. We get an exact sequence

$$
\begin{aligned}
& \underset{n}{\operatorname{colim}}\left[E_{G}^{n \rho_{1}}\left(X / X^{K}: Y\right), \chi\right] \longrightarrow \operatorname{colim}_{n}\left[E_{G}^{n \rho_{1}}(X: Y), \chi\right] \\
& \longrightarrow \operatorname{colim}_{n}\left[E_{G}^{n \rho_{1}}\left(X^{K}: Y\right), \chi\right] \longrightarrow \operatorname{colim}_{n}\left[E_{G}^{n \rho_{1}+1}\left(X / X^{K}: Y\right), \chi\right] .
\end{aligned}
$$

By the above lemma colim $\left[E_{G}^{n \rho_{1}}\left(X / X^{K}: Y\right), \chi\right]=0$ and also colim $\left[E_{G}^{n \rho_{1}+1}(X /\right.$ $\left.\left.X^{K}: Y\right), \chi\right]=0$ replacing $Y$ by $\Sigma Y$. Thus we get the isomorphism

$$
\underset{n}{\operatorname{colim}}\left[E_{G}^{n \rho_{1}}(X: Y), \chi\right] \cong \underset{n}{\operatorname{colim}}\left[E_{G}^{n \rho_{1}}\left(X^{K}: Y\right), \chi\right] \text {. }
$$

Consider the following sequence

$$
\begin{aligned}
& {\left[\Sigma^{m \rho} X^{K}, \Sigma^{n \rho_{1}} E_{m} \wedge Y\right]^{G} \stackrel{\sum_{*}^{n \rho_{2}}}{\longrightarrow}\left[\Sigma^{m \rho+n \rho_{2}} X^{K}, \Sigma^{n \rho} E_{m} \wedge Y\right]^{G}} \\
& \stackrel{\varepsilon_{*}^{n}}{\longrightarrow}\left[\Sigma^{m \rho+n \rho_{2}} X^{K}, E_{n+m} \wedge Y\right]^{G} \stackrel{\Sigma_{*}^{n \rho_{1}}}{\longrightarrow}\left[\Sigma^{(n+m) \rho} X^{K}, \sum^{n \rho_{1}} E_{m+n} \wedge Y\right]^{G}
\end{aligned}
$$

and observe that the composition $=\left(\varepsilon_{*} \circ \Sigma_{*}^{\rho}\right)^{n}$, which proves the isomorphism 


$$
E_{G}^{n \rho_{1}}\left(X^{K}: Y\right) \cong \underset{m}{\operatorname{colim}}\left[\left[\Sigma^{m \rho+n \rho_{2}} X^{K}, E_{n+m} \wedge Y\right]^{G}, \varepsilon_{*} \circ \Sigma_{*}^{\rho}\right] .
$$

And we get the isomorphism

$$
\underset{n}{\operatorname{colim}} E_{G}^{n \rho_{1}}\left(X^{K}: Y\right) \cong \underset{n, m}{\operatorname{colim}}\left[\Sigma^{m \rho+n \rho_{2}} X^{K}, E_{n+m} \wedge Y\right]^{G} .
$$

Observe the commutative diagram:

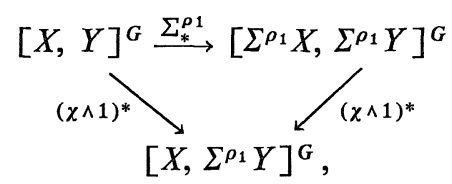

which shows that the homomorphism $\chi$ may be used as $\chi=(\chi \wedge 1)^{*}$ as well as $\chi=(\chi \wedge 1)_{*}$. In the right hand side of the isomorphism (\#\#) we may understand $\chi=(\chi \wedge 1)_{*}$. Then we see that the directed system of this double colimit contains the sequence $\left\{\left[\Sigma^{n \rho_{2}} \wedge X^{K}, E_{n} \wedge Y\right]^{G}, \chi^{\circ} \varepsilon_{*} \circ \Sigma_{*}^{\rho}\right\}$ as a cofinal subsequence. Thus

$$
\underset{n}{\operatorname{colim}} E_{G}^{n \rho_{1}}\left(X^{K}: Y\right) \cong \underset{n}{\operatorname{colim}}\left[\left[\Sigma^{n \rho_{2}} X^{K}, E_{n} \wedge Y\right]^{G}, \chi^{\circ} \varepsilon_{*} \Sigma_{*}^{\rho}\right] \text {. }
$$

Now, $K$ acts trivially on $\sum^{n \rho_{2}} X^{K}$. Hence

$$
\left[\Sigma^{n \rho_{2}} X^{K}, E_{n} \wedge Y\right]^{G}=\left[\Sigma^{n \rho_{2}} X^{K}, E_{n}{ }^{K} \wedge Y^{K}\right]^{G}=\left[\Sigma^{n \rho_{2}} X^{K}, E_{n}{ }^{K} \wedge Y^{K}\right]^{G / K},
$$

and we get the isomorphism

$$
\underset{n}{\operatorname{colim}} E_{G}^{n \rho_{1}}\left(X^{K}: Y\right) \cong\left(\phi_{K} E_{G}\right)^{0}\left(X^{K}: Y^{K}\right),
$$

which, together with $(\#)$, completes the proof of (4.3).

In the exact seqeunce obtained by taking the colimit of (4.1) in the vertical direction, identify one term with $\left(\phi_{K} E_{G}\right)^{\phi_{K^{\alpha}}}\left(X^{K}: Y^{K}\right)$ by (4.3). It is easy to identify colim $\chi^{n}$ with the fixed-point homomorphism $\phi_{K}^{G}$, and we obtain the desired exact sequence

$$
\begin{aligned}
\cdots \longrightarrow\left(\lambda_{K} E_{G}\right)^{\alpha}(X: Y) \longrightarrow E_{G}^{\alpha}(X: Y) \stackrel{\phi_{K}^{G}}{\longrightarrow}\left(\phi_{K} E_{G}\right)^{\phi_{K} \alpha}\left(X^{K}: Y^{K}\right) \\
\longrightarrow\left(\lambda_{K} E_{G}\right)^{\alpha+1}(X: Y) \longrightarrow \cdots
\end{aligned}
$$

for $\alpha \in R O(G)$, which we call the $K$-fixed-point exact sequence.

Let $\pi$ be a set of primes and $H$ a $\pi$-perfect subgroup of $G$. Denote $N$ $=N_{G}(H), W=N_{G}(H) / H, E_{N}=\psi_{N} E_{G}, E_{W}=\phi_{H} E_{N}, \alpha^{\prime}=\psi_{N} \alpha$ and $\alpha^{\prime \prime}=\phi_{H} \alpha^{\prime}$ for $\alpha$ $\in R O(G)$. Consider the following $H$-fixed-point exact sequence (tensored with $\left.\boldsymbol{Z}_{(\pi)}\right)$ :

$$
\cdots \longrightarrow\left(\lambda_{H} E_{N}\right)^{\alpha^{\prime}}(X: Y)_{(\pi)} \longrightarrow E_{N}^{\alpha^{\prime}}(X: Y)_{(\pi)} \stackrel{\phi_{H}^{N}}{\longrightarrow} E_{W}^{\alpha^{\prime \prime}}\left(X^{H}: Y^{H}\right)_{(\pi)} \longrightarrow \cdots
$$


Since actions of $A(N)$ on $E_{N}^{\alpha^{\prime}}(X: Y)$ are natural with respect to $X$ and $Y$, the central idempotent $\bar{e}_{H}^{\pi}$ of $A(N)_{(\pi)}$ acts on this seqeunce as an idempotent. Remark that $\bar{e}_{H}^{\pi}$ acts on $E_{W}^{\alpha^{\prime \prime}}\left(X^{H}: Y^{H}\right)_{(\pi)}$ through the homomorphism

$$
\phi_{H}^{N}: A(N)_{(\pi)} \longrightarrow A(W)_{(\pi)}
$$

defined by $\phi_{H}^{N}[S]=\left[S^{H}\right]$ for finite $N$-sets $S$. By (1.3) we see easily that

$$
\phi_{H}^{N} \bar{e}_{H}^{\pi}=\tilde{e}_{\langle 1\rangle}^{\pi},
$$

the primitive idempotent of $A(W)_{(\pi)}$ corresponding to the trivial $\pi$-perfect subgroup $\{1\}$ of $W$. Thus we get the following exact seqeunce

$$
\begin{aligned}
\cdots \longrightarrow \bar{e}_{H}^{\pi}\left(\lambda_{H} E_{N}\right)^{\alpha^{\prime}}(X: Y)_{(\pi)} & \\
\longrightarrow & \bar{e}_{H}^{\pi} E_{N}^{\alpha^{\prime}}(X: Y)_{(\pi)} \stackrel{\phi_{H}^{N}}{\longrightarrow} \tilde{e}_{\langle 1\rangle}^{\pi} E_{W}^{\alpha^{\prime \prime}}\left(X^{H}: Y^{H}\right)_{(\pi)} \longrightarrow \cdots
\end{aligned}
$$

We will prove

Proposition 4.6. $\bar{e}_{H}^{\pi}\left(\lambda_{H} E_{N}\right)^{\alpha^{\prime}}(X: Y)=0$.

As a corollary of this proposition we obtain

Theorem 4.7. Under the same setting as in Theorem 3.5 there holds the $\bar{e}_{H}^{\pi} A(N)_{(\pi)}$-module isomorphism

$$
\phi_{H}^{N}: \bar{e}_{H}^{\pi} E_{N}^{\alpha^{\prime}}(X: Y)_{(\pi)} \cong \tilde{e}_{\langle 1\rangle}^{\pi} E_{W}^{\phi_{H} \alpha^{\prime}}\left(X^{H}: Y^{H}\right)_{(\pi)},
$$

where $W=N_{G}(H) / H, E_{N}$ is an $N$-spectrum, $E_{W}=\phi_{H} E_{N}, \alpha^{\prime} \in R O(N)$ and $\tilde{e}_{\langle 1\rangle}$ is the primitive idempotent of $A(W)_{(\pi)}$ corresponding to the trivial $\pi$-perfect subgroup $\{1\}$ of $W$.

Proof of Proposition 4.6. Again it is sufficient to prove the special case $\alpha^{\prime}=0 . \quad$ Decompose $\rho_{N}=\rho_{1} \oplus \rho_{2}, \rho_{2}=\rho_{N}^{H} \cong \rho_{W}, \rho_{1}^{H}=\{0\} . \quad$ By (4.2)

$$
\bar{e}_{H}^{\pi}\left(\lambda_{H} E_{N}\right)^{0}(X: Y)_{(\pi)}=\operatorname{colim} \bar{e}_{H}^{\pi} E_{N}^{n \rho_{1}-1}\left(S_{+}^{n \rho_{1}} \wedge X: Y\right)_{(\pi)} .
$$

Therefore it is sufficient to prove

$$
\bar{e}_{H}^{\pi} E_{N}^{0}\left(S_{+}^{n \rho_{1}} \wedge \Sigma X: \Sigma^{n \rho_{1}} Y\right)_{(\pi)}=0 .
$$

Since $\rho_{1}^{H}=\{0\}$, we see that $\left(S^{n \rho_{1}}\right)^{H}=\varnothing . \quad S^{n \rho_{1}}$ is an $N$-CW complex. Let $\sigma^{k}$ $\times N / L$ be an $N$-cell of $S^{n \rho_{1}}$. Then $\left(\sigma^{k} \times N / L\right)^{H}=\sigma^{k} \times(N / L)^{H}=\varnothing$, whence $H \not L L$. The standard argument by induction on $N$-cell of $S_{+}^{n \rho_{1}}$ reduces the problem to show that

$$
\bar{e}_{H}^{\pi} E_{N}^{0}\left(\left(\left(\sigma^{k} \times N / L\right) /\left(\partial \sigma^{k} \times N / L\right)\right) \wedge \Sigma X: \sum^{n \rho_{1}} Y\right)_{(\pi)}=0 .
$$

The left hand side 


$$
\begin{aligned}
& =\bar{e}_{H}^{\pi} E_{N}^{0}\left((N / L)^{+} \wedge \Sigma^{k+1} X: \Sigma^{n \rho_{1}} Y\right)_{(\pi)} \\
& \cong\left(\operatorname{res}_{L}^{N} \bar{e}_{H}^{\pi}\right) \cdot\left(\psi_{L} E_{N}\right)^{0}\left(\Sigma^{k+1} X: \Sigma^{n \rho_{1}} Y\right)_{(\pi)}
\end{aligned}
$$

and

$$
\operatorname{res}_{L}^{N} \bar{e}_{H}^{\pi}=0
$$

by (1.3) as $H \not L L$, which completes the proof.

Apply (3.7) and Theorem 4.7 to the $G$-sphere spectrum. We get the isomorphisms

$$
e_{H}^{\pi} \tilde{\omega}_{G}^{\alpha}(X: Y)_{(\pi)} \cong \bar{e}_{H}^{\pi} \tilde{\omega}_{N}^{\alpha^{\prime}}(X: Y)_{(\pi)} \cong \tilde{e}_{\langle 1\rangle}^{\pi} \tilde{\omega}_{W}^{\alpha^{\prime \prime}}\left(X^{H}: Y^{H}\right)_{(\pi)}
$$

for each $(H) \in P_{\pi}$, where $\alpha \in R O(G), \alpha^{\prime}=\psi_{N} \alpha$ and $\alpha^{\prime \prime}=\phi_{H} \alpha^{\prime}$. Specialize $\pi=\{$ all primes\}, then we get Theorem A (Introduction).

Put

$$
\omega_{G}^{\alpha}(p t)=\tilde{\omega}_{G}^{\alpha}\left(\Sigma^{0}: \Sigma^{0}\right) .
$$

Segal [16] showed the isomorphism

$$
\omega_{G}^{0}(p t) \cong A(G)
$$

Then, by (4.8) we obtain

Corollary 4.9. There hold the ring isomorphisms

$$
e_{H}^{\pi} A(G)_{(\pi)} \cong \bar{e}_{H}^{\pi} A(N)_{(\pi)} \cong \tilde{e}^{\pi}{ }_{1>} A(W)_{(\pi)} .
$$

Specialize Corollary 4.9 to $\pi=\{$ all primes $\}$, then we get Corollary B (Introduction).

Finally we may apply the classifying spaces of families of subgroups due to tom Dieck [6], Let $F_{n \text {-solv }}$ denote the family of all solvable $\pi$-subgroups of $W$ and $E F_{\pi-\text { solv }}$ its classifying space. There holds the isomorphism

$$
\tilde{e}_{\langle 1\rangle}^{\pi} \tilde{\omega}_{W}^{\alpha^{\prime \prime}}\left(X^{H}: Y^{H}\right)_{(\pi)} \cong \tilde{\omega}_{W}^{\alpha^{\prime \prime}}\left(X^{H}: Y^{I I} \wedge E F_{\pi-\varsigma o l v}^{+}\right)_{(\pi)}
$$

by arguments of [6] [7].

Let $H_{0}, H_{1}, \ldots, H_{k}\left(H_{0}=\{1\}\right)$ be a complete system of representatives of $P_{\pi}$. Then, from the direct sum decomposition

$$
\tilde{\omega}_{G}^{\alpha}(X: Y)_{(\pi)}=\coprod_{0 \leqq i \leqq k} e_{H_{i}}^{\pi} \tilde{\omega}_{G}^{\alpha}(X: Y)_{(\pi)}
$$

and (4.8) we get the direct sum decomposition

$$
\tilde{\omega}_{G}^{\alpha}(X: Y)_{(\pi)}=e_{\langle 1}^{\pi}, \tilde{\omega}_{G}^{\alpha}(X: Y)_{(\pi)} \oplus \coprod_{1 \leqq i \leqq k} \tilde{e}_{\langle 1\rangle}^{\pi} \tilde{\omega}_{W_{i}}^{\alpha_{2}}\left(X^{H_{i}}: Y^{H_{i}}\right)_{(\pi)}
$$


where $N_{i}=N_{G}\left(H_{i}\right), W_{i}=N_{i} / H_{i}$ and $\left.\alpha_{i}=\phi_{H_{i}}\left(\psi_{N_{i}} \alpha\right)\right)$ for $1 \leqq i \leqq k$ 。

Example 1. $G=S_{5}, \pi=\{$ all primes $\}$. Conjugacy classes of perfect subgroups are $\left(A_{5}\right)$ and $(\{1\})$, and we obtain the direct sum decomposition

$$
\tilde{\omega}_{G}^{\alpha}(X: Y)=e_{\langle 1\rangle} \tilde{\omega}_{G}^{\alpha}(X: Y)+\tilde{\omega}_{Z / 2}^{\alpha^{\prime \prime}}\left(X^{A_{5}}: Y^{A_{5}}\right),
$$

where $\alpha^{\prime \prime}=\phi_{A_{5}} \alpha$.

Example 2. $G=\mathbb{S}_{6}, \pi=\{$ all primes $\}$. There are 4 conjugacy classes of perfect subgroups: $\left(H_{1}\right),\left(H_{2}\right),\left(H_{3}\right)$ and $\left(H_{4}\right)$, where $H_{1}=A_{6}, H_{2}=A_{5}, H_{3}$ $\cong A_{5}$ and $H_{4}=\{1\} . \quad H_{2}$ and $H_{3}$ are isomorphic but not conjugate. There is an outer automorphism $a$ of $S_{6}$ such that $a\left(H_{2}\right)=H_{3}$. Thus: $N_{G}\left(H_{1}\right)=S_{6}$, $N_{G}\left(H_{2}\right)=S_{5}$ and $N_{G}\left(H_{3}\right) \cong S_{5}$. We obtain the direct sum decomposition

$$
\tilde{\omega}_{G}^{\alpha}(X: Y)=e_{\langle 1\rangle} \tilde{\omega}_{G}^{\alpha}(X: Y) \oplus \mathbb{U}_{1 \leqq i \leqq 3} \tilde{\omega}_{\mathbb{Z} / 2}^{\alpha_{i}}\left(X^{H_{i}}: Y^{H_{i}}\right),
$$

where $\alpha_{i}=\phi_{H_{i}}\left(\psi_{N_{i}} \alpha\right)$ for $1 \leqq i \leqq 3$.

\section{References}

[1] Araki, S. and Murayama, M., $\tau$-cohomology theories, Japan. J. Math., (1978), $363-416$.

[2] — $G$-homotopy types of $G$-complexes and representations of $G$-cohomology theories, Publ. RIMS, Kyoio Univ., $\mathbb{1}$ (1978), 203-222.

[3] Araki, S. and Iriye, $\mathbb{K}$., Equivariant stable homotopy groups of spheres with involutions I, Osaka J. Math., 19 (1982), 1-55.

[4] Bredon, G. E., Equivariant cohomology theories, Eeciure Notes in Math., 3A, SpringerVerlag, 1967.

[5] tom Dieck, T., Lokalisierung äquivarianter Homologie-Theorien, Rfath. Z., $12 \mathbb{1}$ (1971), 253-262.

[6] - Orbittypen und äquivariante Homologie, I, Arch. Math., 23 (1972), 307-317; II, Arch. Math., 26 (1975), 650-662.

[7] - Equivariant homology and Mackey functors, Math. Anit., 206 (1973), $67-78$.

[8] - Transformation groups and representation theory, Leciure Notes in Math., 766, Springer-Verlag, 1979.

[9] Dress, A., A characterization of solvable groups, Math. $Z$., 1 10 (1969), 213-217.

[10] — Contributions to the theory of induced representations, Lecture Notes in Math., 342, Algebraic $K$-theory II, 183-240, Springer-Verlag, 1973.

[11] Gluck, D., Idempotents formula for the Burnside algebra with applications to the psubgroup simplicial complex, Ill. J. Math., 25 (1981), 63-67.

[12] Green, J. A., Axiomatic representation theory for finite groups, J. Pure Appi. Algebra, $\mathbb{1}$ (1971), 41-77.

[13] Kosniowski, C., Equivariant cohomology and stable cohomotopy, Math. Ann., 210 (1974), 83-104. 
[14] Matsumoto, T., Equivariant cohomology theories on G-CW complexes, Osaka $J$. Math., 10 (1973), 51-68.

[15] Nishida, G., The transfer homomorphism in equivariant generalized cohomology theories, J. Math. Kyoto Univ., 18 (1978), 435-451.

[16] Segal, G., Equivariant stable homotopy theory, Actes Congrès Intern. Math., 2 (1970), 59-63.

[17] Yoshida, T., Idempotents of Burnside rings and Dress induction theorem, to appear in J. Algebra. 